\title{
Editorial
}

\section{A word of the Editor-in-Chief}

Restless, uncertain times seem to have arrived. Nevertheless, to rely on trustworthy data sources and common sense is more important than in the calm years of prosperity and ease. We deliver it, hoping that it will bring you a portion of some exciting reading. With a wish of peaceful autumn and a portion of a good mood and health

In Prague, October 28, 2021

Jakub K. Plášil 